\title{
Erratum to: Self-assembled Nanolayers as Interfacial Diffusion Barriers for Thermally Stable and Low Contact Resistance Cu Source/Drain Electrode in $a$-Si:H TFT-LCDs
}

Jung Suk Han, Chiyoung Lee, and Jaegab Lee

DOI: $10.1007 / \mathrm{s} 13391-011-1092-9$

Figure 2 has to be replaced with Fig. 4, Fig. 3 with Fig. 2 and Fig. 4 with Fig. 3. The captions should remain unchanged. 